\title{
ZMIZ1 - A novel Estrogen Receptor co-activator that enhances the growth of ER+ breast cancer
}

\author{
Anže Godicelj ${ }^{\mathrm{a}}$, Ryan Blake ${ }^{\mathrm{a}, \mathrm{e}}$, Federico M Giorgi ${ }^{\mathrm{b}, \mathrm{e}}$, Marcel Gehrunga ${ }^{\mathrm{a}}$, \\ Sanjeev Kumar ${ }^{\mathrm{a}}$, Amy E Cullen ${ }^{\mathrm{a}}$, Kamal Kishore ${ }^{\mathrm{a}}$, Ashley Sawle ${ }^{\mathrm{a}}$, \\ Matthew Eldridge $^{\mathrm{a}}$, Florian Markowetz ${ }^{\mathrm{a}, \mathrm{f}}$, Andrew N Holding,c,d,f \\ ${ }^{a}$ CRUK Cambridge Institute, University of Cambridge, Cambridge, UK \\ ${ }^{b}$ Department of Pharmacy and Biotechnology, University of Bologna, Bologna, Italy \\ ${ }^{c}$ The Alan Turing Institute, 96 Euston Road, Kings Cross, London, UK \\ ${ }^{d}$ Department of Biology, University of York, York YO10 5DD, UK \\ ${ }^{e}$ These authors contributed equally to this work \\ ${ }^{f}$ Corresponding authors
}

\begin{abstract}
The Estrogen Receptor (ER) drives $75 \%$ of breast cancers. On activation, the ER recruits specific co-factors to form a transcriptionally active complex. These co-factors can modulate tumour growth and understanding their roles can help to identify new therapeutic targets.

We applied a quantitative proteomics method, qPLEX-RIME , to analyse the ER protein complex and characterise changes in protein-protein interactions on activation. Our analysis identified ZMIZ1 as novel co-factor within the ER chromatin-bound complex, extending its known role as a co-factor of the Androgen Receptor. We find further evidence for an ER-ZMIZ1 interaction by showing that both proteins are co-expressed in biopsy samples. We characterise ZMIZ1 function by showing that targeting ZMIZ1 results in the reduction of ER transcriptional activity and significantly reduces the proliferation of ER-positive cell lines. We validated these results genome-wide by RNA-seq and identified that targeting ZMIZ1 resulted in a specific reduction of estradiol-induced cell cycle genes.

These results establish ZMIZ1 as a having a key role in the ability of the ER to activate key genes that drive the proliferation of breast cancer, and its biological importance in patient tumours.
\end{abstract}

Keywords: Estrogen Receptor, ZMIZ1, Breast Cancer, Cancer, Co-factors, Transcription, Nuclear Receptors, Signalling, Prostate Cancer, Patient 


\section{Outcome}

\section{Introduction}

Breast cancer is the most common form of cancer in women worldwide. Stratification of the disease into multiple sub-types improved patient out4 comes by targeting subtype-specific drivers [11].

5 ER positive breast cancer. Approximately $75 \%$ of breast cancers are classified as Estrogen Receptor (ER) positive. In these cancers, the ER is no longer correctly regulated, subverts cell division regulation, and becomes the driving transcription factor in the tumour [16]. Only a few primary breast cancers have mutations in the ER [19], yet the transcriptional activity is frequently abnormal [3]. In many metastatic tumours the ER is still active and drives the growth of the tumour with a reduced dependence on estrogen or in a ligand-independent manner [25,31]. This critical role for the ER in disease progression has therefore made the protein a key target for therapeutics like Fulvestrant [20] and Tamoxifen [10]. More recently, Tamoxifen has been prescribed as a preventive treatment in high-risk healthy patients to successfully reduce their chances of developing breast cancer [6]. Most women benefit from endocrine therapy with $50-70 \%$ of cases responding to treatment. However, relapse is very common with the risk ranging from 10 to $41 \%$ [21].

Co-factors of the ER. One strategy to overcome relapse focusses on co-factors of the ER. The majority of ER binding sites are at distal enhancer elements [4]. On binding to these sites the receptor catalyses the formation of chromatin loops and recruits several co-activators along with the mediator complex. It is through these interactions that the ER is able to facilitate the activation of RNA Pol II at the promoters of target genes [17]. Without the coordination of the ER and these co-factors it therefore is not possible for the efficient transcription of target genes to occur.

Further characterisation of ER co-factors could therefore be essential to identify targets for novel treatment strategies. Successful examples of cofactor based approaches include studies demonstrating that the GREB1-EREZH2 transcriptional axis is directly involved in tamoxifen resistance [35] or identifying the pioneer-factor FOXA1 as a key opportunity for future interventions $[18,2]$. This study aims to lay the ground work for future cofactor based therapies by presenting proteomic and genomic evidence that 
ZMIZ1 is co-expressed with ER in patient tumours and that the inhibition in ZMIZ1 reduces the ability of the ER to promote the cell cycle within ER+ breast cancer tumour cells.

\section{Results}

\section{1. $q P L E X-R I M E$ of ER activation identifies novel co-factor ZMIZ1}

We used qPLEX-RIME, a state-of-the-art method for quantifying proteinprotein interactions within nuclear receptor complexes [22], to quantitatively monitor the changes in protein-protein interactions in the ER complex after activating it with estradiol at 0 and 45 minutes across four isogenic replicates (Figure 1A).

Detected protein-ER interactions that were both found to significantly change on stimulation with estradiol $(\mathrm{p}<0.05)$ and had a two-fold change in protein intensity were reviewed for known biology. We found several previously identified ER co-factors including RARA, CBP, EP300, NRIP1 and GATA3. We also detected SUMO1-3 within the ER complex, most likely as a result of the covalent modification of the estrogen receptor or another protein within the ER chromatin-bound complex, in agreement with previous ChIP-seq experiments [32]. In addition, we also found putative novel protein partners of ER (Figure 1B).

The list of potential new ER cofactors includes ZMIZ1 (Figure 1C), which had previously been identified as a co-activator of the Androgen Receptor (AR) $[15,28]$. However, despite activating AR, transfection of ZMIZ1 into CV-1 cells was not able to enhance GR, PR, ER, and VDR-mediated transcription [28]. This result has since been incorporated in the GeneCards and Uniprot databases [33]. Unpublished yeast data, discussed by [28], supports the conclusion that there is no interaction between ZMIZ1 and non-AR nuclear receptors.

Given that our qPLEX-RIME data shows a significant interaction between ER and ZMIZ1 in the breast cancer setting, and that we did not detect the presence of $\mathrm{AR}$, we considered the breast cancer specific function of ZMIZ1 to be of key interest for follow-up studies.

\subsection{ZMIZ1 knock-down reduces ER transcriptional activity}

We hypothesised that as our breast cancer model was able to support the ER-ZMIZ1 interaction that ZMIZ1 may have a transcriptional role, in the ER complex. 


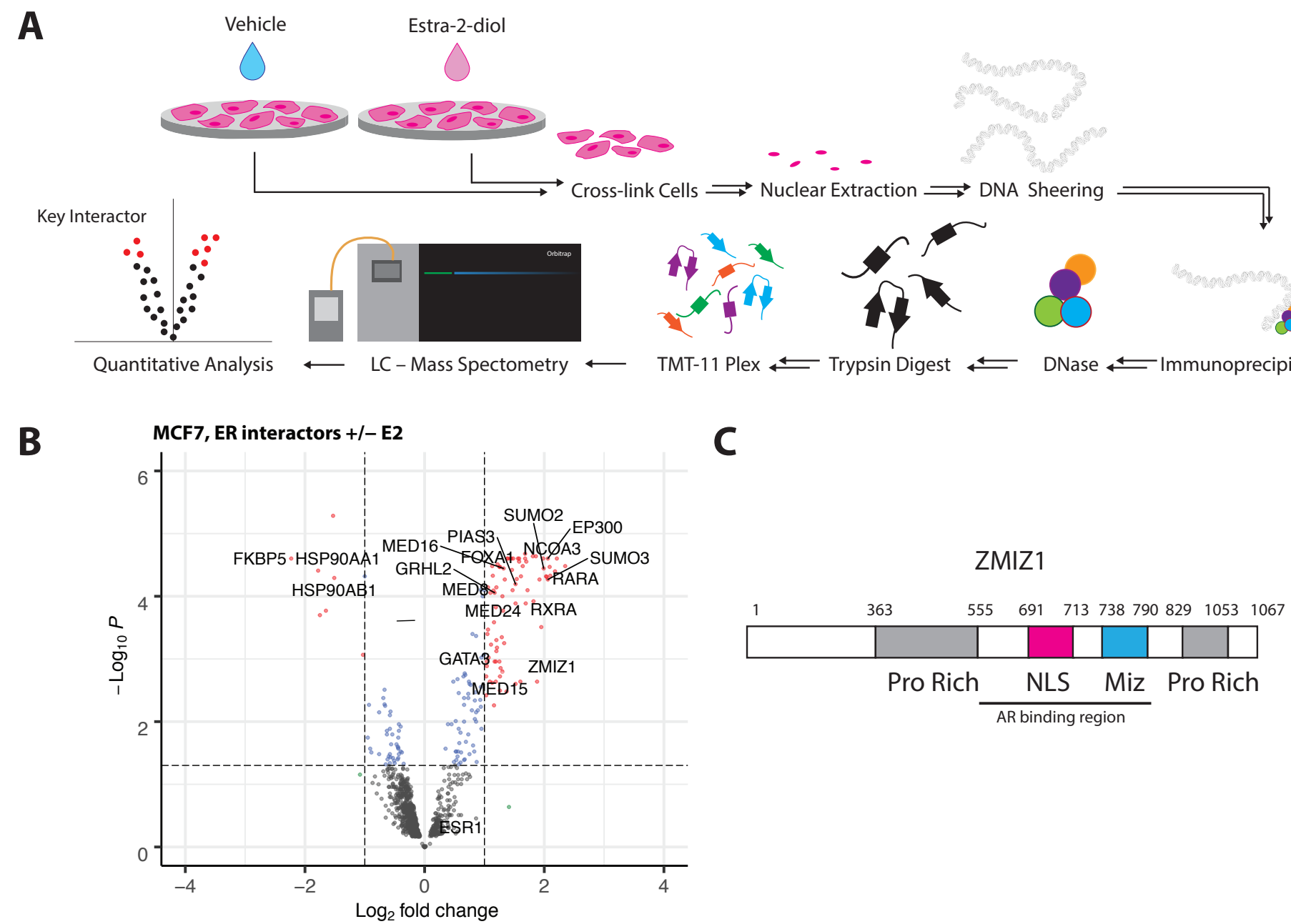

Figure 1: (A) qPLEX-RIME enables the quantitative comparision of multiple conditions to idenfity key interactions between transcription factors on the chromatin. We stimulated ER+ breast cancer cell lines with estradiol and undertook a comparative analysis against an unstimulated control to identified the changes in the ER interacome on activation. (B) ER interacting proteins identified by qPLEX-RIME in MCF7. Top ranking differentialassociated proteins detected with $\mathrm{P}<0.05$ and $\operatorname{LogFC}>1$ are highlighted in red. Gain of known co-factors GATA3 [30], RARA[27], EP300, GRHL2 [8] and NCOA3 were all detected along with the loss of HSP90 on binding of estradiol by the ER. Parts of the mediator complex: MED8, MED16, and MED24, along with pioneer factor FOXA1, were also detected. ZMIZ1 has not previously been reported to interact with the ER. (C) A schematic presentation of the ZMIZ1 protein. The AR binding region was previously identified by the interaction of a 556-790aa truncated mutant with a AR-GAL4 DBD fusion protein leading to activation of $\beta$-gal reporter gene. The C-terminal, proline-rich region of ZMIZ1, was identified as an intrinsic trans-activation domain (TAD)[28]. 
To determine if ZMIZ1's role within the ER complex regulated transcriptional activity we monitored the changes in ER activity on knockdown of ZMIZ1 with siRNA using a Cignal Reporter Assay. The activity of the ER was monitored in two models of ER-positive breast cancer, MCF7 and T47D, along with MDA-MB-231, a model of Triple Negative Breast Cancer. Estrogen receptor activity was measured by a luciferase activity assay relative to a constitutive active positive control. Renilla luciferase activity was used to control for transaction efficiency.

Both MCF7 and T47D (Figure 2A) cell lines showed significantly reduced ER activity $(\mathrm{P}<0.044$ and $\mathrm{P}<0.0045$ respectively, paired t-test, onetailed, $\mathrm{n}=4$ ). As expected, MDA-BM-231 showed no detectable ER activity, and therefore no significant difference between the siZMIZ1 and siCTRL conditions or between the experimental conditions and the negative control. These results confirm that ZMIZ1's role is specific to the ER+ breast cancer sub-types and that expression of the ER was essential for the effect seen.

\subsection{ZMIZ1 knock-down delays response to E2 in ER regulated genes}

Since knockdown of ZMIZ1 leads to a significant reduction in ER transcriptional activity we hypothesised that knockdown of ZMIZ1 would also result in a reduced response to E2 at downstream targets of the ER.

To test this hypothesis we set up a paired RNA-seq experiment treating MCF7 cells with either siZMIZ1 or siCTRL. In both cases we undertook four isogenic replicates, measuring transcriptional levels at 3, 6, 12 and 24 hours after stimulation with estradiol.

Between the siZMIZ1 and control condition, the largest number of differentially expressed genes occurred at 6 hours after stimulation with E2 (30 hours after initial knockdown). In contrast, by 24 hours (48 hours after knockdown of ZMIZ1) only 14 genes were detected as differentially expressed.

Analysis of the 6 hour time-point (siZMIZ1 vs siCTRL) by Gene Set Enrichment Analyis (GSEA)[29] for enrichment of ER responsive genes gave conflicting results. We identified three published gene sets relevant to the cell culture models used: STEIN_ESR1_TARGETS and BHAT_ESR1_TARGETS_ NOT_VIA_AKT1_UP described the genes regulated by the ER in the MCF7 cell line, while WILLIAMS_ESR1_TARGETS_UP reflected genes activated on the stimulation of the ER with estradiol in the T47D cell line. GSEA analysis of the differential expression at 6 hours of stimulation with E2 in MCF7 for STEIN_ESR1_TARGETS gave a non-significant reduction $(\mathrm{p}=0.07)$, BHAT ESR1_TARGETS_NOT_VIA_AKT1_UP gave a significant increase $(\mathrm{p}=0.003)$, and 
A

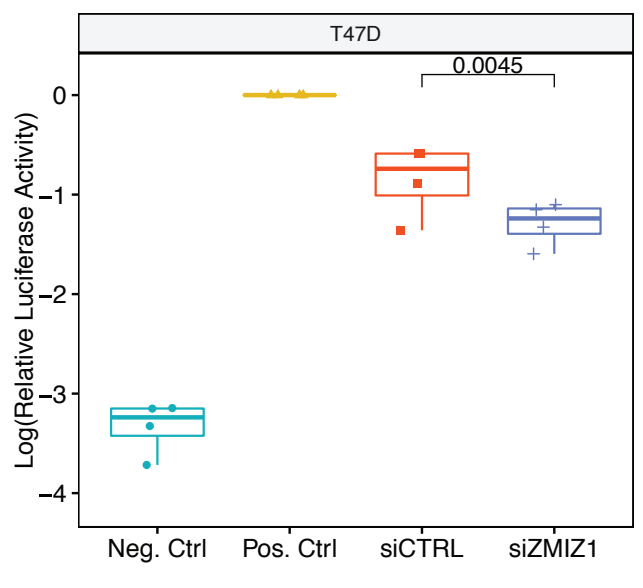

B

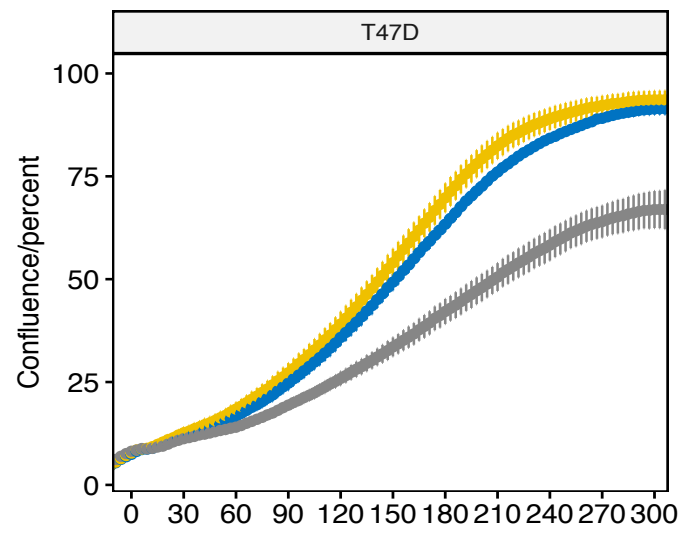

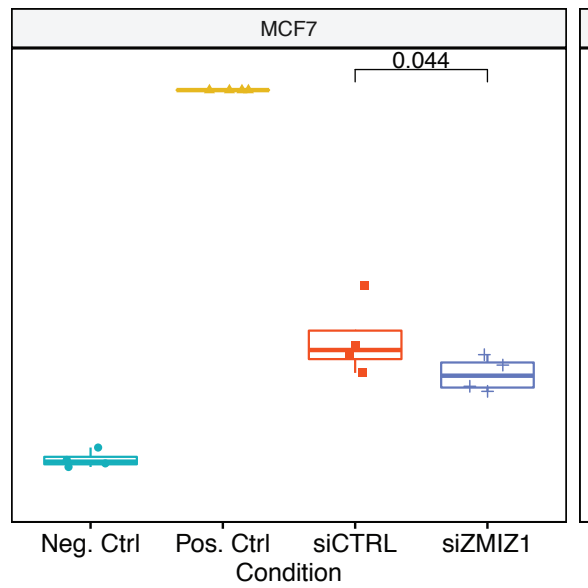

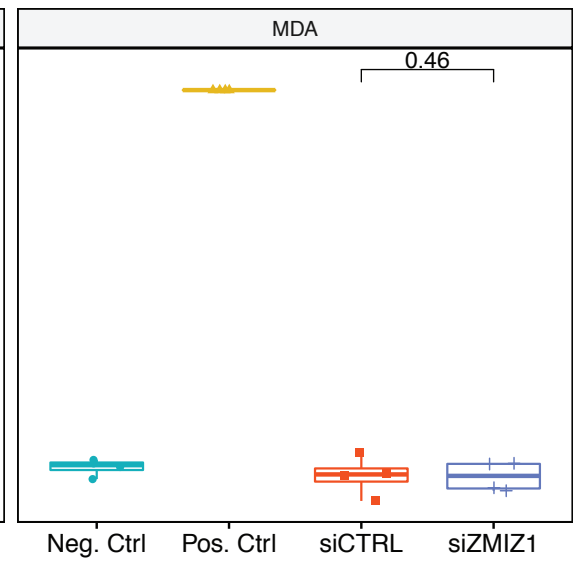

Condition $\rightarrow$ RNAiMAX $\rightarrow$ siCTRL $\rightarrow$ siZMIZ1
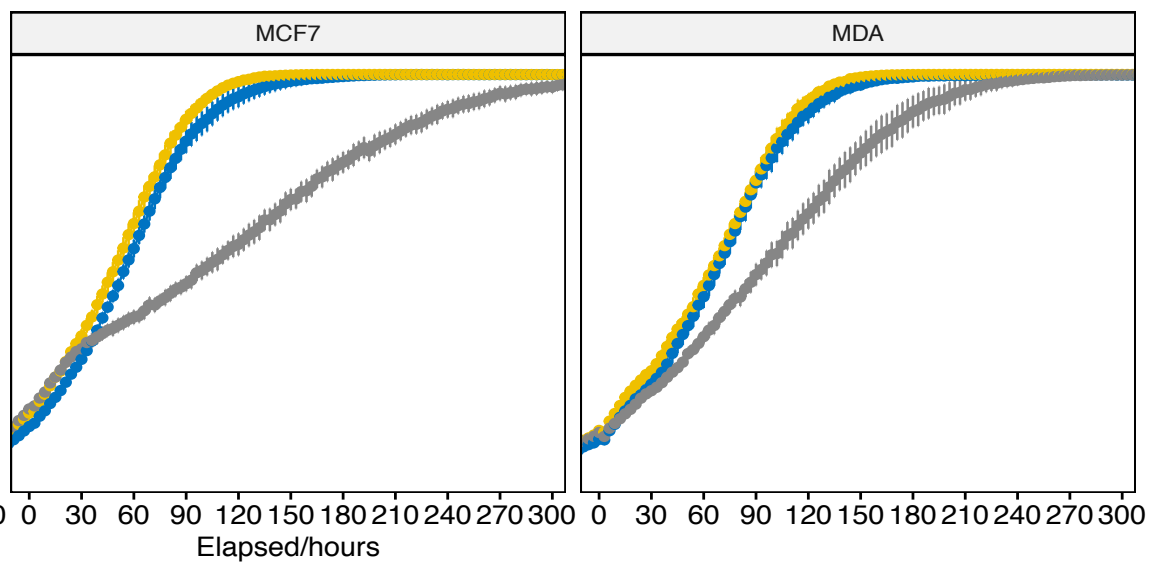

Figure 2: (A) Lucierase Assay monitoring ER activity. ER activity in MCF7, T47D and MDA-MB-231 cell lines was monitored by Cignal ERE Reporter Assay. Both MCF7 and T47D cells showed a significant reduction in ER activity in response to ZMIZ1 knockdown. As expected, MDA-MB-231 cells showed no activity in all conditions but the positive, constitutively active, control.(B) Knockdown of ZMIZ1 in three cell lines, T47D, MCF7 and MDA-MB-231 all showed reduced proliferation. The effect was largest in the ER-postive cell lines, T47D and MCF7. We inferred the increased response to ZMIZ1 knockdown to imply that as ER singalling is the main driver in these two cell lines that ZMIZ1 expression levers had the greatest effect. In contrast the response in MDA-MB-231, a triple-negative breast cancer model, implies that ZMIZ1 has additional functionality independent of the estrogen receptor transcriptional complex, but this has a lesser effect on cell growth than its role in ER positive breast cancer. 
WILLIAMS_ESR1_TARGETS_UP showed a significant reduction in ER transcriptional response $(\mathrm{p}=0.047)$. Overall overlap of all 3 gene sets is low with 3 genes in common. The STEIN_ESR1_TARGETS gene set has $25 \%$ overlap with the other two sets, while the WILLIAMS_ESR1_TARGETS_UP gene set, and BHAT_ESR1_TARGETS_NOT_VIA_AKT1_UP have $46 \%$ and $9 \%$ overlap respectively with the rest of the genes sets Given the ER's role in regulating cell cycle [9], we undertook GSEA against three MSigDB gene sets [29] focused on cell cycle: GO_CELL_CYCLE, KEGG_CELL_CYCLE, and REACTOME_CELL_CYCLE. All three gene sets gave a significant results $\left(\mathrm{p}=2.3 \times 10^{-6}, 0.0001 \& 3.5\right.$ $\times 10^{-7}$ respectively).

On the basis of these results, we hypothesised that ZMIZ1 regulated a subset of ER-regulated genes focused on cell cycle. The varied results for GSEA of ER specific sets could then be explained as a result in variation in genes represented in each of MSigDB gene sets and a lack of specificity to ERZMIZ1 signalling axis. To test our hypothesis were undertook GSEA against the intersection of the REACTOME_CELL_CYCLE and the previously tested ER specific gene sets $(\mathrm{p}=0.0006)$. Repeating the analysis in T47D cell gave $\mathrm{p}$ $=0.005$ (Figure $\mathrm{S} 1)$.

\subsection{ZMIZ1 knock-down reduces proliferation of breast cancer cell lines}

On the basis of these results, we tested the hypothesis that knockdown of the ZMIZ1 protein would result in reduced proliferation of ER-positive cancer cell line models.

Analysis of two ER-positive (MCF7 and T47D) and one triple negative breast cancer model (MDA-MB-231) showed that knockdown of ZMIZ1 reduced cell proliferation in all three cell lines. The effect was greatest in the ER positive cell-lines, T47D and MCF7, with T47D growth reaching a maximum at a reduced confluence compared to the control experiments. In contrast, the magnitude of the reduction in growth was more modest in the MDA-MB-231 cell line and the rate of recovery was much higher. The susceptibility of T47D and MCF7 to ZMIZ1 knockdown is likely due to the specific reliance of these cells on ER for cell growth, while in the MDA-MB-231 cell line ZMIZ1 likely regulates a second ER-independent pathway (Figure 2B).

\subsection{ZMIZ1 and ER activities correlate in patient samples}

If ZMIZ1 is a key component of the ER complex, we would expect its activity to be higher in ER-positive than in ER-negative breast cancer. To test this hypothesis, we used two large gene expression collections, TCGA [34] 
and METABRIC [5], to assess co-expression of ER and ZMIZ1 in a patient setting. In both data sets, ZMIZ1 had significantly increased expression in luminal over basal sub-types (TCGA: $\mathrm{p}=1.34 \times 10^{-23}$, Wilcoxon test, $\mathrm{n}=962$; METABRIC: $\mathrm{p}=1.18 \times 10^{-10}$, Wilcoxon text, $\left.\mathrm{n}=961\right)$.

To address if both ZMIZ1 and ER proteins were transcriptionally active within patient samples we then generated regulatory network models for both the TCGA and METABRIC data sets using ARACNe-AP[12]. Using these networks, we applied the VIPER algorithm[1] to calculate the activity of ER and ZMIZ1 proteins in both patient sample data-sets (Figure 3B). For both TCGA and METABRIC we saw a significant correlation $\left(\mathrm{p}=4.77 \times 10^{-85}\right.$ and $\mathrm{p}=1.02 \times 10^{-139}$, respectively) between the activity of the two transcription factors, with greatest activity of both TF networks in the luminal sub-type.

\subsection{ZMIZ1 and ER are co-localised in patient samples}

To further validate the link between ER and ZMIZ1, we checked if ZMIZ1 expression was co-localised with that of the ER in clinical material. Visual inspection of ER-positive breast cancer tumours from three patients showed strong nuclear staining of both proteins in adjacent sections. Comparison of the localisation of staining between ER and ZMIZ1 demonstrated that both proteins were found within the nucleus of epithelial cells, and that infiltrating cells were absent for both of the proteins. Further, the distribution of ER and ZMIZ1 staining correlated, suggesting the ER and ZMIZ1 are expressed within the same cells of the patient tumours (Figure 4).

\subsection{High ZMIZ1 expression correlates with low survival in ER+ patients}

To explore if the role of ZMIZ1 activity held clinical importance we investigated if ZMIZ1 expression was a predictor of patient survival in the context of both ER-positive and ER-negative tumours using KMplot[13].

Stratifying ER-positive patients by median ZMIZ1 expression showed that ER-positive patients with higher levels of ZMIZ1 expression had significantly poorer outcome $(\mathrm{P}=0.0023$, Logrank test). In contrast, the same analysis of ER-negative patients demonstrated no significant difference in patient outcome $(\mathrm{P}=0.49$, Logrank test, Figure $3 \mathrm{~A})$.

These results were consistent with the ZMIZ1 functioning as a co-factor of the ER in ER-positive breast cancer and that ZMIZ1 played a role in disease progression in ER-positive breast cancer. 
A

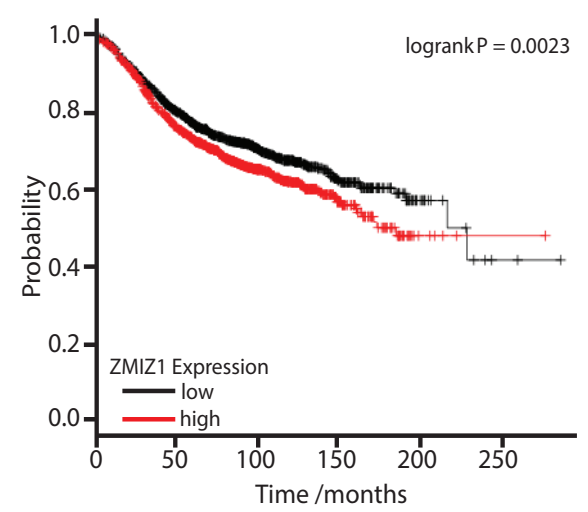

B

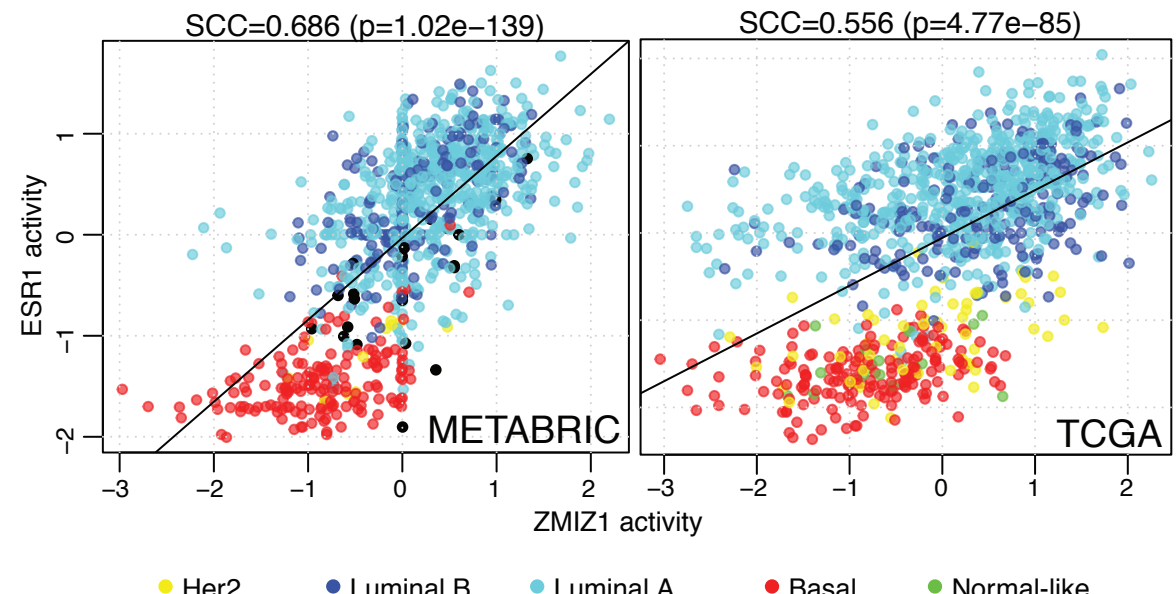

Figure 3: (A) Stratifying patients of ER+ (left) breast cancer based on ZMIZ1 expression (median cut-off) shows that high levels of ZMIZ1 results in a significant reduction in patient survival. The effect is not seen with ER -ve (right) breast cancer, implying an ER+ specific function for ZMIZ1 in breast cancer. (B) ER positive patient tumour biopsies stained for ER and ZMIZ1. ZMIZ1 is found localised in the nucleus of cells that show a similar distribution as ER+ cells within the tumour, while the infiltrating cells from the host are found absent for both ER and ZMIZ1. 


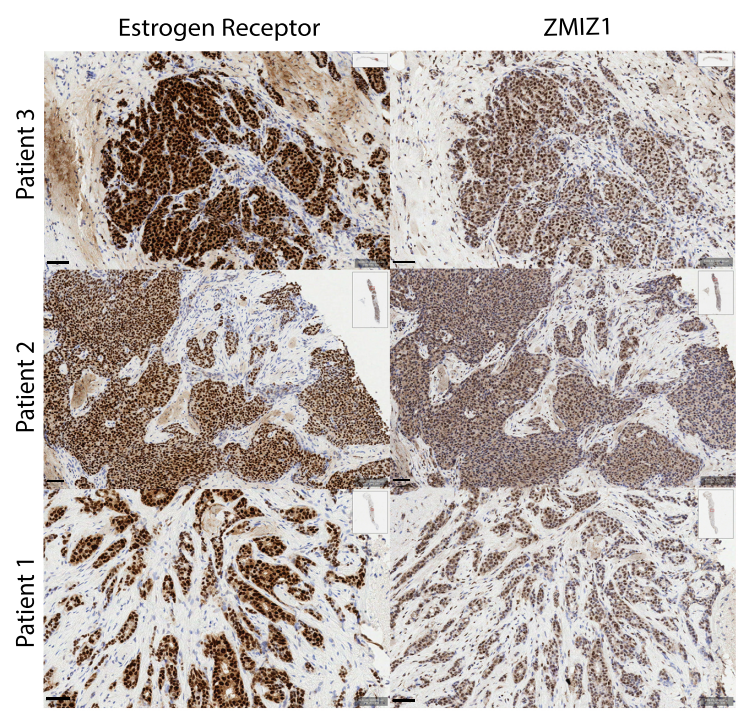

Figure 4: Consecutive slides derived from biopsy samples of three patients with ER+ breast cancer were stained by IHC for both the Estrogen Receptor-alpha and ZMIZ1. In all three patients the localisation of staining for the proteins was specific to the tumour cells. In contrast, the stromal cells show little or no expression of either protein.

\section{Discussion}

ZMIZ1 is a known co-activator of the androgen receptor (AR) [28] and interacts with numerous transcription factors and DNA binding proteins, including NOTCH1 [24], and p53 [14]. ZMIZ1 is related to Protein Inhibitor of Activated STAT (PIAS) proteins and contains a highly conserved MIZ/SPRING zinc finger domain (Figure 1C) [24, 28], which is involved in mediating protein-protein interactions. Knockdown of ZMIZ1 in a prostate cancer cell line has been shown to reduce proliferation [23] with ZMIZ1-mediated coactivation of AR by either direct or indirect SUMOylation of the receptor [28]. The similarities in the roles of AR in prostate and ER in breast cancer, along with evidence that SUMOylation plays an import role in ER biology [32], suggest that a similar mechanism could exist in ER-postive breast cancer

Our analysis in both the MCF7 and T47D cell lines (ER-positive breast cancer models) shows a significant change in ER-mediated transcriptional activity on ZMIZ1 knockdown. We therefore present evidence that ZMIZ1 has a role in modulating ER activated transcription that has not previously been seen in other models. Further, we were able to show that within patient 
samples the ZMIZ1 and ER expression correlated, and that the proteins are co-localised with in the nuclei of tumour cells.

In this study, we demonstrate that ZMIZ1 is able to interact with the ER complex and that loss of ZMIZ1 results in lower transcriptional activity. Previously it has been shown that ZMIZ1 has a very strong trans-activation domain (TAD) [28] and our study that is consistent with this mechanism, suggesting that ZMIZ1 is a co-activator within the ER complex. Alternatively, ZMIZ1 may activate the ER by promoting the SUMOylation of either the ER or its co-factors. A similar role has been seen for the AR, increasing SUMOylation by about 40\% [28], and our own qPLEX-RIME analysis of the ER shows increase identified SUMO protein modifications on stimulation estradiol. However, the mechanism by which ZMIZ1 can promote SUMOylation remains unclear.

All nuclear receptors orchestrate their response on activation by interactions with large numbers of co-regulators [26]. Loss or gain of function mutations that effect these co-factors may then result in a selective advantage for the tumour, or even lead to resistance to treatment [35]. In this context, ZMIZ1's role in mediating AR driven cell growth [28] has led to suggestions that the protein holds an interesting opportunity for targeting prostate cancer disease progression during androgen deprivation therapy[15]. In breast cancer the ER's role in driving cell growth and proliferation is a key factor in patient survival and levels of ZMIZ1 are a significant predictor of patient outcome. These features of ZMIZ1 suggest similar potential for targeting the protein as a therapeutic opportunity limiting ER-positive breast cancer growth. This hypothesis is further supported that our analysis of the transcriptome after knockdown of ZMIZ1 showed a specific and significant reduction in estrogen-mediated cell cycle gene activation.

In conclusion, further studies into the molecular basis by which ZMIZ1 regulates both ER and AR mediated transcription may provide new insight into the biological role of ZMIZ1 in hormone driven cancers along with novel opportunities for therapeutic intervention.

\section{Experimental Procedures}

\section{$q P L E X-R I M E$}

qPLEX-RIME samples were prepared as previously reported [22]. Cells were grown in estrogen-free culture as previously described [7] and crosslinked at 45 minutes after addition of $100 \mathrm{nM}$ E2 or ethanol. 
ERE activity Assay

Four biological replicates of MCF7, T47D and MDA-MB-231 were assayed using Cignal ERE Reporter Assay Kits (Qiagen) according to the manufacture protocol. Estrogen-free culture was established as previously reported[7]. SMARTpool ZMIZ1 and control siRNA (Dharmacon) were transfected with Lipofectamine RNAiMAX Transfection Reagent (Thermo Fisher Scientific) following the manufactures protocol.

\section{Cell Growth Assay}

MCF7, T47D and MDA-MB-231 cell growth was monitored by incucyte. siRNA knockdown was undertaken with SMARTpool on-target siZMIZ1 along with the matched siCTRL (Dharmacon) and was transfected with Lipofectamine RNAiMAX reagent (Thermo Fisher Scientific) according to manufactures protocol. Cells were reverse transected, media was refreshed after 24 hours. Knockdown was confirmed by RT-qPCR.

\section{Immunohistochemistry}

De-waxing and re-hydration prior to IHC automated on the Leica ST5020, along with the post-IHC de-hydration and clearing. Sections mounted using Leicas coverslipper CV5030. Samples were run on Leicas Polymer Refine Detection System (DS9800) using their standard template on the automated Bond-III platform. The specific antibody targeting ZMIZ1 was purchased from R\&D Systems (AF8107) and used at a concentration of $2 \mu \mathrm{g} / \mathrm{ml}$. The sodium citrate pre-treatment is run at $100{ }^{\circ} \mathrm{C}$. The secondary (post primary) was rabbit anti-sheep from Jackson ImmunoResearch (r313-005-003), diluted 1:500. DAB Enhancer is included as an ancillary reagent (Leica, AR9432). All samples are from the PIONEER trial, REC number 17/NE/0113.

\section{$R N A-S e q$}

Estrogen-free culture was established as previously reported[7]. SMARTpool ZMIZ1 and control siRNA (Dharmacon) were purchased and transfected with Lipofectamine RNAiMAX Transfection Reagent (Thermo Fisher Scientific) following the manufactures protocol 24 hours prior to stimulation with 100nM E2 or ethanol control. Media was refreshed before stimulation with E2 to minimise toxicity of the transaction reagent. RNA-seq samples were prepared using TruSeq RNA Library Prep Kit v2 (illumina) according to the manufactures protocol. Libraries were sequenced using the Illumina HiSeq 4000 platform, and aligned with Hisat v 2.1.0. The GSEA implementation used was from the VULCAN R-package [8]. 


\section{Data and code availability}

All RNA-seq data have been deposited in the GEO database with the accession number GSE133381. The code and data to generate Figures 1B, 2A, 2B, 3B and Figure S1 is available as an R-package from https://github.com/andrewholding/ZMIZ1.

\section{Acknowledgments}

We would like to acknowledge the support of The University of Cambridge and Cancer Research UK.

This work was funded by CRUK core grant C14303/A17197 and A19274 (to FM) and supported by The Alan Turing Institute under the EPSRC grant EP/N510129/129/1 as a Turing Fellowship to ANH.

We would like to acknowledge the contribution from the CRUK Genomics, Proteomics, Histopathology/ISH, and Bioinformatics core facilities in supporting this work.

\section{Author Contributions}

AG, RB, AEC and ANH undertook the experimentation. SK provided patient material. AG and SK generated ZMIZ1 stained images of patient samples. AG, RB, KK, AS, ME, FMG and ANH analyzed the data. MG analysed the ZMIZ1 stained images of patient samples. FMG undertook network analysis of TGCA and METABRIC data-sets. AG and ANH designed the experiments. AG, ANH, FM wrote the manuscript. All authors were involved in the proofing and editing of drafts.

\section{Declaration of Interests}

None Declared.

\section{References}

[1] M. J. Alvarez, Y. Shen, F. M. Giorgi, A. Lachmann, B. B. Ding, B. H. Ye, \& A. Califano (2016). Functional characterization of somatic mutations in cancer using network-based inference of protein activity. Nat. Genet., $48,838-847$. 
[2] F. M. Behan, F. Iorio, G. Picco, E. Goncalves, C. M. Beaver, G. Migliardi, R. Santos, Y. Rao, F. Sassi, M. Pinnelli, et al. (2019). Prioritization of cancer therapeutic targets using CRISPR-Cas9 screens. Nature, .

[3] J. Carroll (2016). Eje prize 2016: Mechanisms of estrogen receptor (er) gene regulation in breast cancer. European journal of endocrinology, (pp. EJE-16).

[4] J. S. Carroll, X. S. Liu, A. S. Brodsky, W. Li, C. A. Meyer, A. J. Szary, J. Eeckhoute, W. Shao, E. V. Hestermann, T. R. Geistlinger, et al. (2005). Chromosome-wide mapping of estrogen receptor binding reveals long-range regulation requiring the forkhead protein FoxA1. Cell, 122, 33-43.

[5] C. Curtis, S. P. Shah, S. F. Chin, G. Turashvili, O. M. Rueda, M. J. Dunning, D. Speed, A. G. Lynch, S. Samarajiwa, Y. Yuan, et al. (2012). The genomic and transcriptomic architecture of 2,000 breast tumours reveals novel subgroups. Nature, 486, 346-352.

[6] J. Cuzick, I. Sestak, S. Cawthorn, H. Hamed, K. Holli, A. Howell, J. F. Forbes, E. Abdi, E. Anderson, C. Atkinson, et al. (2015). Tamoxifen for prevention of breast cancer: extended long-term follow-up of the IBIS-I breast cancer prevention trial. Lancet Oncol., 16, 67-75.

[7] M. J. Guertin, A. E. Cullen, F. Markowetz, \& A. N. Holding (2018). Parallel factor ChIP provides essential internal control for quantitative differential ChIP-seq. Nucleic Acids Res., 46, e75.

[8] A. N. Holding, F. M. Giorgi, A. Donnelly, A. E. Cullen, S. Nagarajan, L. A. Selth, \& F. Markowetz (2019). VULCAN integrates ChIP-seq with patient-derived co-expression networks to identify GRHL2 as a key co-regulator of ERa at enhancers in breast cancer. Genome Biol., 20, 91.

[9] S. JavanMoghadam, Z. Weihua, K. K. Hunt, \& K. Keyomarsi (2016). Estrogen receptor alpha is cell cycle-regulated and regulates the cell cycle in a ligand-dependent fashion. Cell Cycle, 15, 1579-1590.

[10] V. C. Jordan (2003). Tamoxifen: a most unlikely pioneering medicine. Nat Rev Drug Discov, 2, 205-213. 
[11] D. C. Koboldt, R. S. Fulton, M. D. McLellan, H. Schmidt, J. KalickiVeizer, J. F. McMichael, L. L. Fulton, D. J. Dooling, L. Ding, E. R. Mardis, et al. (2012). Comprehensive molecular portraits of human breast tumours. Nature, 490, 61-70.

[12] A. Lachmann, F. M. Giorgi, G. Lopez, \& A. Califano (2016). ARACNeAP: gene network reverse engineering through adaptive partitioning inference of mutual information. Bioinformatics, 32, 2233-2235.

[13] A. Lanczky, A. Nagy, G. Bottai, G. Munkacsy, A. Szabo, L. Santarpia, \& B. Gyorffy (2016). miRpower: a web-tool to validate survival-associated miRNAs utilizing expression data from 2178 breast cancer patients. Breast Cancer Res. Treat., 160, 439-446.

[14] J. Lee, J. Beliakoff, \& Z. Sun (2007). The novel PIAS-like protein hZimp10 is a transcriptional co-activator of the p53 tumor suppressor. Nucleic Acids Res., 35, 4523-4534.

[15] X. Li, C. Zhu, W. H. Tu, N. Yang, H. Qin, \& Z. Sun (2011). ZMIZ1 preferably enhances the transcriptional activity of androgen receptor with short polyglutamine tract. PLoS ONE, 6, e25040.

[16] H. Mohammed, C. D'Santos, A. A. Serandour, H. R. Ali, G. D. Brown, A. Atkins, O. M. Rueda, K. A. Holmes, V. Theodorou, J. L. Robinson, et al. (2013). Endogenous purification reveals GREB1 as a key estrogen receptor regulatory factor. Cell Rep, 3, 342-349.

[17] S. Murakami, A. Nagari, \& W. L. Kraus (2017). Dynamic assembly and activation of estrogen receptor enhancers through coregulator switching. Genes Dev., 31, 1535-1548.

[18] H. Nakshatri \& S. Badve (2007). FOXA1 as a therapeutic target for breast cancer. Expert Opin. Ther. Targets, 11, 507-514.

[19] S. Oesterreich \& N. E. Davidson (2013). The search for ESR1 mutations in breast cancer. Nat. Genet., 45, 1415-1416.

[20] C. K. Osborne, A. Wakeling, \& R. I. Nicholson (2004). Fulvestrant: an oestrogen receptor antagonist with a novel mechanism of action. $\mathrm{Br}$. J. Cancer, 90 Suppl 1, 2-6. 
[21] H. Pan, R. Gray, J. Braybrooke, C. Davies, C. Taylor, P. McGale, R. Peto, K. I. Pritchard, J. Bergh, M. Dowsett, et al. (2017). 20-Year Risks of Breast-Cancer Recurrence after Stopping Endocrine Therapy at 5 Years. N. Engl. J. Med., 377, 1836-1846.

[22] E. K. Papachristou, K. Kishore, A. N. Holding, K. Harvey, T. I. Roumeliotis, C. S. R. Chilamakuri, S. Omarjee, K. M. Chia, A. Swarbrick, E. Lim, et al. (2018). A quantitative mass spectrometry-based approach to monitor the dynamics of endogenous chromatin-associated protein complexes. Nat Commun, 9, 2311.

[23] Y. Peng, J. Lee, C. Zhu, \& Z. Sun (2010). A novel role for protein inhibitor of activated STAT (PIAS) proteins in modulating the activity of Zimp7, a novel PIAS-like protein, in androgen receptor-mediated transcription. J. Biol. Chem., 285, 11465-11475.

[24] N. Pinnell, R. Yan, H. J. Cho, T. Keeley, M. J. Murai, Y. Liu, A. S. Alarcon, J. Qin, Q. Wang, R. Kuick, et al. (2015). The PIAS-like Coactivator Zmiz1 Is a Direct and Selective Cofactor of Notch1 in T Cell Development and Leukemia. Immunity, 43, 870-883.

[25] D. R. Robinson, Y. M. Wu, P. Vats, F. Su, R. J. Lonigro, X. Cao, S. Kalyana-Sundaram, R. Wang, Y. Ning, L. Hodges, et al. (2013). Activating ESR1 mutations in hormone-resistant metastatic breast cancer. Nat. Genet., 45, 1446-1451.

[26] M. G. Rosenfeld \& C. K. Glass (2001). Coregulator codes of transcriptional regulation by nuclear receptors. J. Biol. Chem., 276, 3686536868 .

[27] C. S. Ross-Innes, R. Stark, K. A. Holmes, D. Schmidt, C. Spyrou, R. Russell, C. E. Massie, S. L. Vowler, M. Eldridge, \& J. S. Carroll (2010). Cooperative interaction between retinoic acid receptor-alpha and estrogen receptor in breast cancer. Genes Dev., 24, 171-182.

[28] M. Sharma, X. Li, Y. Wang, M. Zarnegar, C. Y. Huang, J. J. Palvimo, B. Lim, \& Z. Sun (2003). hZimp10 is an androgen receptor co-activator and forms a complex with SUMO-1 at replication foci. EMBO J., 22, 6101-6114. 
[29] A. Subramanian, P. Tamayo, V. K. Mootha, S. Mukherjee, B. L. Ebert, M. A. Gillette, A. Paulovich, S. L. Pomeroy, T. R. Golub, E. S. Lander, et al. (2005). Gene set enrichment analysis: a knowledge-based approach for interpreting genome-wide expression profiles. Proc. Natl. Acad. Sci. U.S.A., 102, 15545-15550.

[30] V. Theodorou, R. Stark, S. Menon, \& J. S. Carroll (2013). GATA3 acts upstream of FOXA1 in mediating ESR1 binding by shaping enhancer accessibility. Genome Res., 23, 12-22.

[31] W. Toy, Y. Shen, H. Won, B. Green, R. A. Sakr, M. Will, Z. Li, K. Gala, S. Fanning, T. A. King, et al. (2013). ESR1 ligand-binding domain mutations in hormone-resistant breast cancer. Nat. Genet., 45, 14391445 .

[32] T. Traboulsi, M. El Ezzy, V. Dumeaux, E. Audemard, \& S. Mader (2018). Role of SUMOylation in differential ER transcriptional repression by tamoxifen and fulvestrant in breast cancer cells. Oncogene, .

[33] UniProt Consortium. (2019). UniProt: a worldwide hub of protein knowledge. Nucleic Acids Res., 47, D506-D515.

[34] J. N. Weinstein, E. A. Collisson, G. B. Mills, K. R. Shaw, B. A. Ozenberger, K. Ellrott, I. Shmulevich, C. Sander, J. M. Stuart, K. Chang, et al. (2013). The Cancer Genome Atlas Pan-Cancer analysis project. Nat. Genet., 45, 1113-1120.

[35] Y. Wu, Z. Zhang, M. E. Cenciarini, C. J. Proietti, M. Amasino, T. Hong, M. Yang, Y. Liao, H. C. Chiang, V. G. Kaklamani, et al. (2018). Tamoxifen Resistance in Breast Cancer Is Regulated by the EZH2-ER-GREB1 Transcriptional Axis. Cancer Res., 78, 671-684.

\section{Supplimentry Figures}


bioRxiv preprint doi: https://doi.org/10.1101/789610; this version posted October 2 2019. The copyright holder for this preprint (which was not certified by peer review) is the author/funder, who has granted bioRxiv a license to display the preprint in perpetuity. It is made available under aCC-BY-NC 4.0 International license.
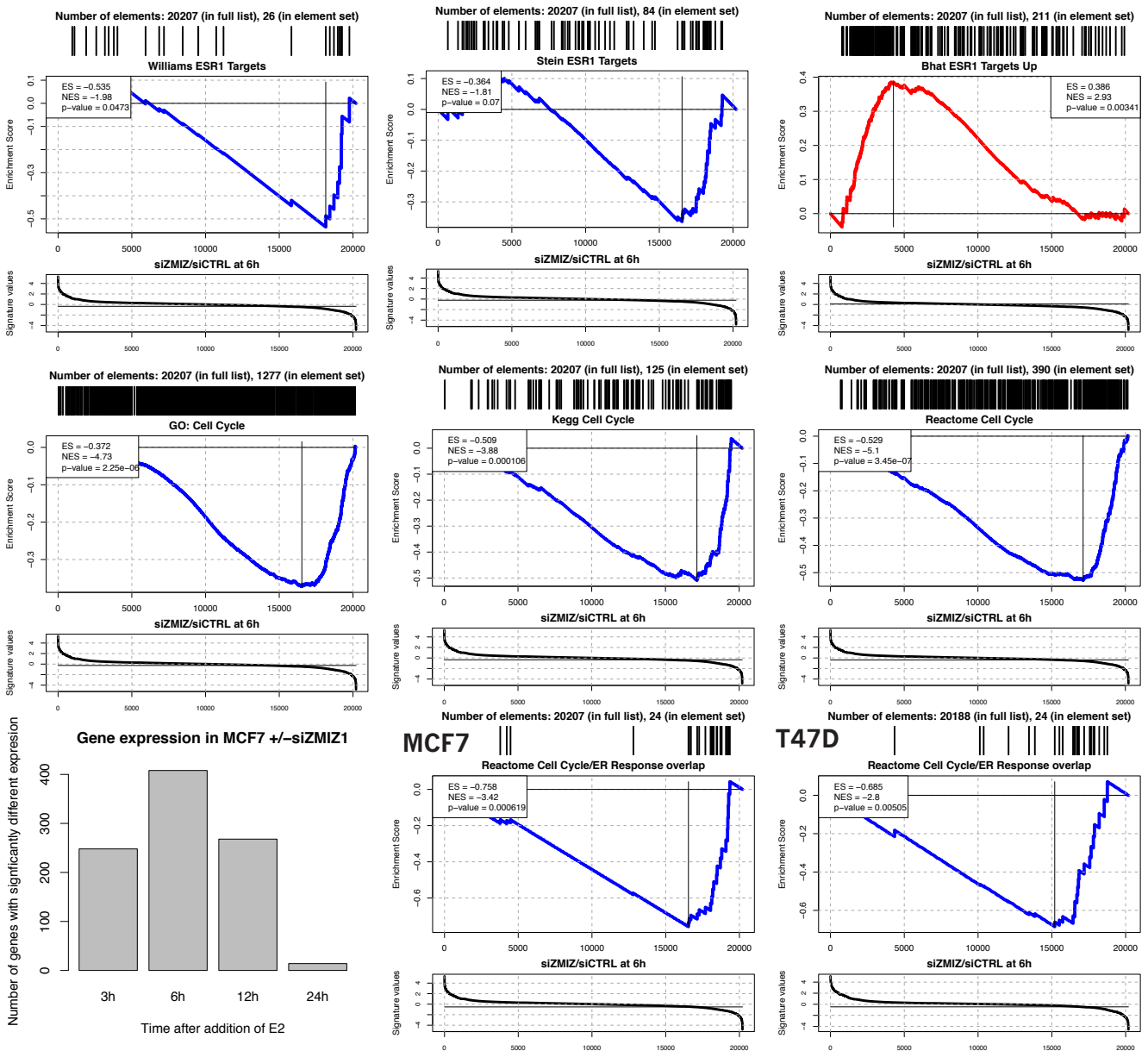

Figure S1: GSEA analysis of RNA-seq +/-siZMIZ1. Row 1-2, GSEA of differentially expressed genes +/-siZMIZ1 at 6 hours after addition of E2. Row 3, (Left) Number of differentially expressed genes +/-siZMIZ1 at 3, 6, 12 and 24 hours after addition of E2. (Middle and Right) GSEA of cell cycle specific ER responsive genes shows knockdown of ZMIZ1 targets these genes. 